\title{
A Multiphysics Model of Capillary Growth and Remodeling
}

\author{
Dominik Szczerba ${ }^{1}$, Gábor Székely ${ }^{1}$, and Haymo Kurz ${ }^{2}$ \\ ${ }^{1}$ Computer Vision Lab, ETH, CH-8092 Zürich, Switzerland \\ ${ }^{2}$ Institute of Anatomy and Cell Biology, University of Freiburg, D-79104 Freiburg, Germany
}

\begin{abstract}
We report on an enhanced computational framework for simulating flow-tissue interactions that significantly expands the capabilities of our previous model [1]. We adhere to the basic structural concept of the so-called intussusceptive growth and remodeling which does not only generate capillaries and terminal vessels but also rebuilds them into a highly perfused system [2]. Present enhancements comprise calculation and visualization in three dimensions, refined tissue and fluid mechanics, and the transport of molecules that act as biochemical growth or signaling factors. Our present model explains formation of capillary meshes and bifurcations, and the emergence of feeding and draining microvessels in an interdigitating pattern that avoids arterio-venous shunts. In addition, it predicts detailed hydrodynamic properties and transport characteristics for oxygen, metabolites or signaling molecules. In comparison to the previous work, the complexity of our approach is dramatically increased by using a multiphysics modeling environment, where many independent computational components are combined and the data structure is unified.
\end{abstract}

\section{Introduction}

The long-standing interest of biology and medicine in a thorough quantitative understanding of the vascular system and its development has gained new impetus due to increased efficiency of computational resources during the past two decades. Different aspects of normal and tumor angiogenesis have been subject to in silico modeling, and such different approaches have been chosen as fractal self-similar constructs [3], cellular automata [4], functional macroscopic pipelines ([5], [6], [7], [8]), optimal design principles [9] and various mathematical formulations ([10], [11], [12]). A more comprehensive review of available literature can be found in our previous publications ([6], [7], [1]) and will not be repeated here. Although many of those models are successful in predicting structurally realistic systems with relevant biophysical properties, they do not demonstrate fundamental understanding of how such structures actually come into existence from the microscopic point of view. Such understanding is, however, mandatory if realistic simulations of, e.g., anti-angiogenic cancer treatments or the effects of irradiation are considered.

In our previous approach, formation of blood vessels was treated as flow-driven remodeling of a planar capillary network or plexus demonstrated to be present at many anatomical sites. The tissue was represented as a set of discrete entities (pixels) displacing under shear stress determined by the local flow conditions. The model successfully 
predicted bifurcation formation and micro-vessel separation from a capillary bed, but failed to ensure correct topology of the resulting arterial and venous branches, thus producing shunt vessels.

We therefore attempted to implement cellular response mechanisms that would result in self-avoidance of arterial and venous branches. We found that the interplay of production and transport of molecular compounds controlling the development, refined tissue and fluid dynamics (including non-linear material responses) and remodeling into the third dimension result in self-avoidance and interdigitating patterns of the terminal branches. This allowed us to achieve better similarity to real patters and hence improved predictive power.

Fundamental differences between other models that we are aware of and our current approach are: 1) addressing each and every single capillary loop and terminal arterial or venous branch - not just vector fields or concentration gradients, or only ramified trees; 2) using exclusively local interactions - without explicitly enforcing global optimality or a priori prescribing bifurcation parameters. We thus hope to demonstrate that the emergence of gross functional optimality in macroscopically tree-like networks can be realized by molecular mechanisms that act on the microscopic scale. Major innovations in this mathematically and computationally challenging project are presented here.

\section{Numerical Background}

Most generally spoken, we base our modeling on in vivo observations (cf. cited references) that strongly justify mechanistic assumptions about vascular tissues that are able to respond to hydrodynamic conditions and signaling molecules by shape changes. Numerically, the core of our model underlying all computations is a differential form of the general transport equation for property $\phi$, usefully written in the following form:

$$
\frac{\partial \rho \phi}{\partial t}+\operatorname{div}(\rho \phi \boldsymbol{u})=\operatorname{div}(\operatorname{\Gamma grad} \phi)+S_{\phi},
$$

with $\phi$ denoting the transported property, $\Gamma$ diffusivity, $\rho$ density, $\boldsymbol{u}$ convective vector field and $S_{\phi}$ being the sources (or sinks) of the transported property.

Invoking the Gauss theorem and integrating over a three dimensional control volume $V$ yields an integral (conservative) equation for the transported property:

$$
\frac{\partial}{\partial t} \int_{V} \rho \phi d V+\int_{A}(\rho \phi \boldsymbol{u}) \boldsymbol{d a}=\int_{A}(\Gamma \operatorname{grad} \phi) \boldsymbol{d} \boldsymbol{a}+\int_{V} S_{\phi} d V,
$$

The above finite volume discretization is further linearized and written as:

$$
A_{P}^{\phi} \phi_{P}=A_{i}^{\phi} \phi_{i}+S_{P}^{\phi}
$$

with summation over explicit cell neighbours and compass notation as in [13]. The above equation has to hold true for every point in the grid. This can be formulated as a linear problem $A x=b$, where $x$ is a vector of unknown scalar values, $A$ is a domaindependent (and property-specific) coefficient matrix and $b$ is given by the boundary conditions and the solution from the previous time steps. 
The above sketched general computational technique allows to address a wide range of transport phenomena by setting $\phi$ to an implicit geometry function, velocity components, concentrations, internal energy, temperature, etc. and identifying the diffusive coefficient accordingly (mass diffusivity, fluid viscosity, etc.).

\section{Tissue Modeling}

Simulation of biological phenomena most often requires dealing with complex geometries. Unstructured body-fitted meshes, while offering obvious advantages like delivering accurate solutions at the boundaries, are computationally too expensive in case of complex anatomical domains. Therefore, in the presented model we rely on Cartesian grids, eventually using adaptive refining, as seen in Fig. 1 .

We represent the tissue formally as a mathematical continuum, separated from external fluid (blood) by an interface defined in a level set sense. An introduction to this family of methods is beyond the scope of this report and can be found elsewhere [14]. Here we only briefly discuss the most important issues relevant to our modeling. The key ingredient is to define an implicit or embedding function to represent the fluid-structure interface to be tracked. In our case it clearly will be the deforming blood-tissue interface. Deforming such an interface is computationally equivalent to solving a transport problem with $\phi=\phi_{l s}$ and $\boldsymbol{u}$ being an interface advancing vector field. Such a convenient formulation offers a natural treatment of topological changes like break-up and fusion of the boundaries, however, does not provide explicit geometrical information about the domain. In particular, this means that we will not be able to (directly) describe local geometry or topology as no geometric primitives as pipes or tripods will be available. On the other hand, a big advantage of this technique is that the level set function is continuous and the associated partial differential equations can be handled with a common, previously introduced numerical method. Such a treatment allows to drive the domain remodeling in an elegant manner using deformation fields (available near the boundaries) instead of arbitrary displacements introduced in our previous work [1].
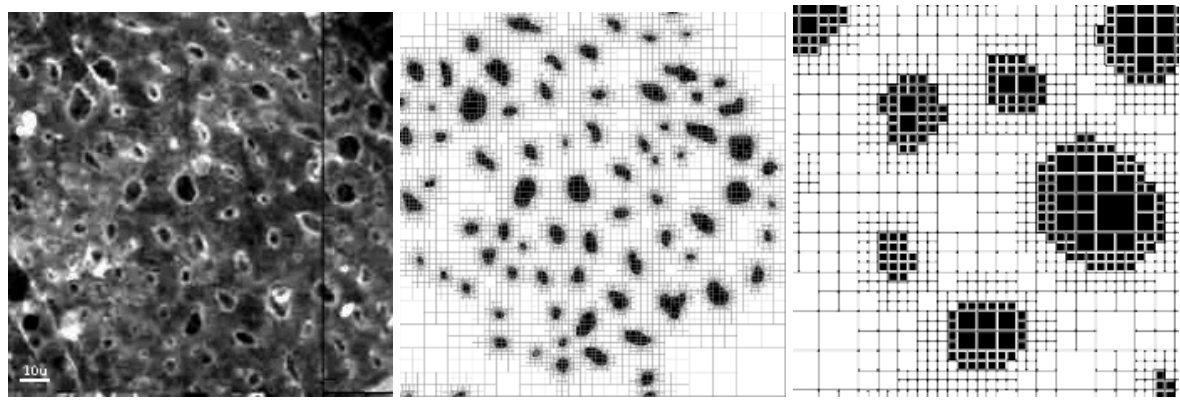

Fig. 1. A close-up of an initial input (left) for the simulations along with computational grid demonstrating adaptive refinement (middle) to the blood-tissue boundary in a real capillary plexus. Note the mesh inside the black tissue pillars (right): the discretization of the whole domain is needed to solve the level-set equation. 
Whereas previously we only considered the tangential shear stress as the remodeling factor, using the current formulation we are able to derive the deformation fields generated by any sources, including boundary viscous and pressure forces, surface tension and gradients of growth factors. In addition, we propose a non-linear tissue response to the shear stress of the form $f(\tau) \sim 1 /\left(\tau+\tau_{0}\right)$. This means that due to decreased cellular sensitivity the tissue will deform less to increasingly stronger stimulus exerted by the flowing fluid.

\section{Flow Conditions}

In order to provide hemodynamic conditions in the domain of interest, the NavierStokes equations governing the fluid flow have to be solved. Generally speaking, this can be done by using the general transport equation (taking $\phi=u, v, w$ ), relating the source terms to the pressure gradient and enforcing the mass-conservation equation (taking $\phi=1$ ) - and additionally neglecting the rate of change of $\rho$ for an incompressible fluid. Unfortunately, such treatment will not guarantee smooth pressure solution, as pressure will not be present in the resulting mass-conservation equation. Solving such a system will result in oscillatory pressure solutions, therefore this case needs special treatment. We chose a recent smoothing pressure correction approach [15], which unlike most other established techniques, allows calculations to be performed using a colocated variable arrangement. The whole solution procedure results in discrete distributions of velocity and pressure in the domain of interest, which can be used to derive any further variables like, e.g., shear stress, experimentally known to modulate endothelial cell remodeling via realignment and elongation [2]. To control the remodeling process using flow derived forces we first calculate the viscous stress tensor $\tau$ :

$$
\tau=\eta\left(\nabla \boldsymbol{u}+(\nabla \boldsymbol{u})^{T}\right)
$$

In addition to the stress due to viscous forces, there can also be internal stresses due to the fluid pressure:

$$
\sigma=-p \boldsymbol{I}
$$

where $I$ is the identity matrix. From these stress tensors, the viscous and pressure boundary forces (normal, tangential) per unit area can be derived and directly added to the interface advancing vector field displacing the tissue boundary. Another component of this field can be surface tension:

$$
\sigma_{s}=\sigma_{0} \kappa \boldsymbol{n} \delta
$$

where $\sigma_{0}$ is a material coefficient, $\kappa$ denotes the curvature, $\boldsymbol{n}$ is an interface normal vector and $\delta$ is a delta function of the implicit level-set function (non-zero only at the zero level-set). We also attempt to include some approximations of the known nonlinear properties of blood. As a first order approximation to the experimentally known shear-thinning behaviour of blood we pick a power law fluid, where the apparent or effective viscosity is a function of the shear rate as given by:

$$
\eta^{*}=K D^{n-1},
$$


where $D$ is the shear rate (independent of fluid orientation) and $K, n$ are the model parameters to be found experimentally. More sophisticated versions of such power law rheology are also available in our model to simulate the non-newtonian regime of the flow, however, they are all only valid for homogeneous fluids. In capillaries with a few microns diameter, however, blood does not fulfill this assumption. In particular, the finite sizes of the red blood cells could lead to explicit momentum or molecular transfer to the walls during collisions or cause mechanical obstructions in thinner capillaries. We do not currently address these effects in our model.

\section{Transport Properties}

We regard the mechanical response of a tissue exposed to physical influence of external flow to be a major factor in capillary plexus growth. It is, however, not yet understood, how molecular signaling interacts with this process and how these two effects can be integrated within a single comprehensive model of vascular tissue morphogenesis. Once the computational method is available to solve the general transport equation it becomes straight-forward to model mass transport of any chemical molecules like oxygen, carbon dioxide or growth factors. Technically speaking, nothing restricts us from introducing a few such molecular partners and allowing them to react with each other. First order chemical reactions can easily be modeled by modifications of the corresponding source terms, e.g.:

$$
\begin{array}{cc}
S_{\mathrm{O}_{2}}=-\theta_{\mathrm{O}_{2}} \phi_{\mathrm{O}_{2}}, & \text { (burning oxygen) } \\
S_{C O_{2}}=-S_{\mathrm{O}_{2}}, & \text { (into carbon dioxide) } \\
S_{A G F}=\theta_{A G F} \phi_{C O_{2}}, \text { (producing growth factors in ischemic areas) } \\
S_{l s}=\theta_{l s} \phi_{A G F}, & \text { (enforcing/inhibiting tissue remodeling) }
\end{array}
$$

etc. with $\phi_{x}$ relevant properties, $S_{x}$ production/consumption rates (positive/negative), and $\theta_{x}$ local tissue activities. Modeling possibilities here are limited solely by experimental knowledge.

\section{Results}

The simulation workflow is composed of all the components described so far. First, initial structural conditions are derived experimentally from confocal microscopy images of an early developmental stage of chicken embryo capillary plexus. The resulting images are enhanced and segmented using standard, semi-automatic image processing techniques. Binarized blood-tissue boundaries become the input for the flow simulation, thereby determining hemodynamic conditions in the domain. Currently, the input is two-dimensional, which is a sufficient approximation of the initial stage of a real capillary plexus. The tissue remodeling during the simulation, however, is permitted into a thin three-dimensional layer (see [2] for background information about the real remodeling process). The next version of our framework, however, is planned to use full 3-D segmentation for structural input. 


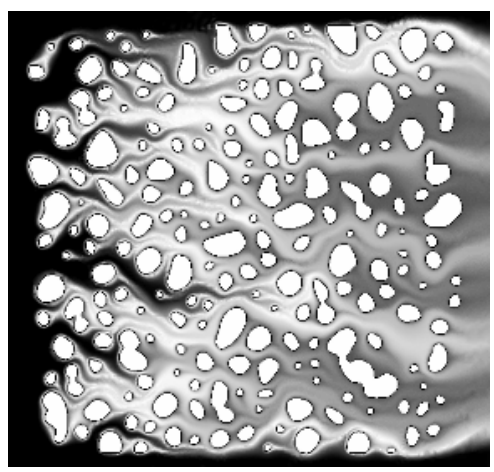

Fig. 2. Simulation of transport from the arterial (left) to the venous (right) side of a respiratory capillary plexus. Oxygen is diffused into the domain through the top face of the square image area and then convected with the flowing blood. In response to its time-averaged exposure, a heavy growth factor is produced and also convected with the flowing fluid. Color intensity values correspond to its concentration levels and the white areas correspond to the tissue pillars (black in Fig. 11. Note the inhomogeneous concentration that results from greatly varying passage times through the capillary plexus, which can in itself represent a growth or remodeling signal.
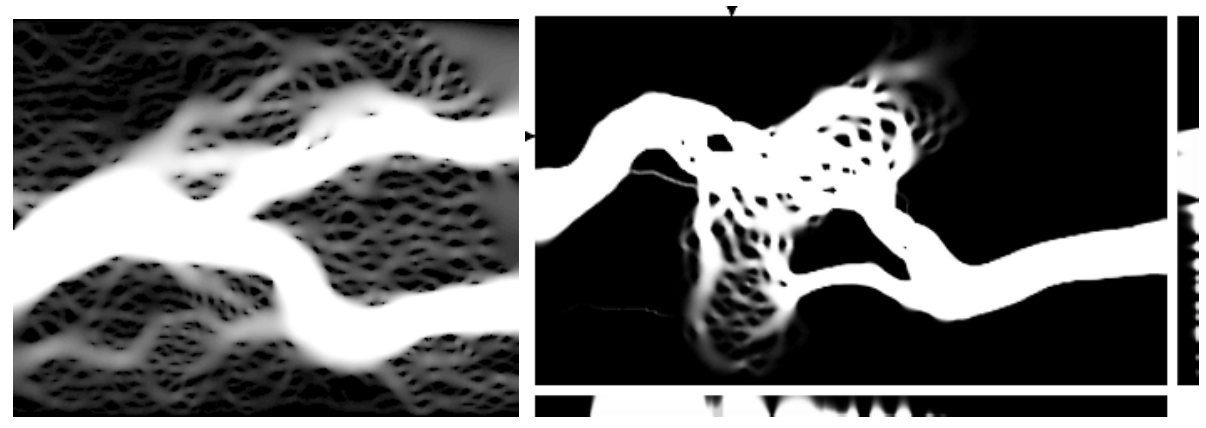

Fig. 3. Final stage of a virtual vascular development. Left: a 2-D network. Due to linear tissue response and lack of chemical signalling a shunt is formed. Right: a 3-D network. Formation of an arterio-venous shunt is avoided and a primitive capillary exchange plexus is formed in between the feeding artery (left) and draining vein (right). At the right and bottom sides, cross-sections of the structure are shown. Both results originate from the same input geometry. Size of the 3-D grid: $600 \times 400 \times 20$.

Second, the global deformation field is computed, and molecular components $\left(\mathrm{O}_{2}\right.$, $\mathrm{CO}_{2}$, growth factor) are dissolved and eventually allowed to react with the tissue surface (Fig. 2). The resulting vector field is transferred to the tissue simulation module, where shape changes are calculated as physical deformations (including surface tensions). Thus, the solution determined for a given input domain is used for structural remodeling of the entire system. This procedure is iterated in subsequent steps for a preset time period or until steady-state is reached. Note, that opposed to our previous 2-D approach, we do not anymore attempt to a priori separate between the experimentally known sub-modes of the intussusceptive remodeling. Instead, we presume they all 
should emerge as a result of the simulations once a sufficient level of modeling complexity is reached. In particular, we do not explicitly insert new pillars into low shear stress regions; instead, we observe their natural appearance from adjacent layers in 3-D.

A possible stable result of remodeling an initially uniform capillary plexus into a differentiated microvascular network via our multiphysics simulation is shown in Fig.3(right). Obviously, higher order vessels are formed that avoid direct fusion (arteriovenous shunt) by interdigitation: one feeder artery aims between two draining venous branches. This will not be the case if our previous remodeling procedure is applied Fig. 3 (left). We identified chemical signalling in 3-D as well as a non-linear tissue response to strain as the keys to avoiding the malformations. The non-linear nature of blood, at least in the homogeneous regime we address, is not mandatory to achieve these results and can be accounted for in the tissue response function. This will noticeably increase the performance without seriously compromising the modeling efficiency. It could also be noted that the simulation process itself has reached a considerable level of complexity. Even if it is still far away from inherent complexity of real vascular networks, however, it excels most other models that we are aware of in this aspect, hopefully leading to superior predictive power. While we are currently evaluating the performance of our multiphysics framework with respect to robustness and similarity to real microvessels, this work is beyond the scope of this article.

\section{Conclusions}

The findings resulting from our multiphysics multiscale model are consistent with observations on real morphology, where a microvascular tree is generated from a capillary plexus. In addition to explaining the formation of bifurcations and the separation of microvessels, our new method is also able to predict a primitive interdigitation of the terminal branches and avoids arterio-venous malformations. Moreover, the current version allows computational studies about the transport of oxygen, metabolites or signaling molecules. Our results demonstrate that interdisciplinary multicomponent computer models of blood vessel networks can integrate experimental data on the cellular level to simulate supracellular morphogenesis with unprecedented detail.

\section{References}

1. Szczerba, D., Székely, G.: Computational model of flow-tissue interactions in intussusceptive angiogenesis. Journal of Theoretical Biology 234(1) (2005) 87-97

2. Kurz, H., Burri, P., Djonov, V.: Angiogenesis and vascular remodeling by intussusception: From form to function. News in Physiological Sciences 18 (2003) 65-70

3. Lindenmayer, A.: Mathematical models for cellular interaction in development. Journal of Theoretical Biology 18 (1968) 280-315

4. Alarcon, T., Byrne, H., Maini, P.: A cellular automaton model for tumour growth in inhomogeneous environment. Journal of Theoretical Biology 225 (2003) 257-274

5. Sandau, K., Kurz, H.: Modelling of vascular growth processes: a stochastic biophysical approach to embryonic angiogenesis. Journal of Microscopy 175 (1994) 205-213

6. Gödde, R., Kurz, H.: Structural and biophysical simulation of angiogenesis and vascular remodeling. Developmental Dynamics 220 (2001) 387-401 
7. Szczerba, D., Székely, G.: Macroscopic modeling of vascular systems. In: Medical Image Computing and Computer-Assisted Intervention - MICCAI 2002. Volume 2489 of Lecture Notes in Computer Science., LNCS Springer (2002) 284-292

8. Szczerba, D., Székely, G.: Simulating vascular systems in arbitrary anatomies. In: Medical Image Computing and Computer-Assisted Intervention - MICCAI 2005: 8th International Conference, Palm Springs, CA, USA,. Volume 3750 of Lecture Notes in Computer Science., Springer-Verlag GmbH (2005) 641-648

9. Schreiner, W., Buxbaum, P.F.: Computer optimization of vascular trees. IEEE Transactions on Biomedical Engineering 40 (1993) 482-491

10. Baish, J.W., Gazit, Y., Berk, D.A., Nozue, M., Baxter, L.T., Jain, R.K.: Role of tumor vascular architecture in nutrient and drug delivery: An invasion percolation-based network model. Microvascular Research 51 (1996) 327-346

11. Anderson, A., Chaplain, M.: Continuous and discrete mathematical models of tumor-induced angiogenesis. Bulletin of Mathematical Biology 60 (1998) 857-899

12. McDougall, S.R., Anderson, A.R.A., Chaplain, M.A.J., Sherratt, J.A.: Mathematical modelling of flow through vascular networks: Implications for tumour-induced angiogenesis and chemotherapy strategies. Bulletin of Mathematical Biology 64 (2002) 673-702

13. Ferziger, J., Perić, M.: Computational Methods for Fluid Dynamics. Springer-Verlag (2002)

14. Sethian, J.A.: Level Set Methods and Fast Marching Methods. Cambridge University Press (1999)

15. Date, A.W.: Solution of transport equations on unstructured meshes with cell-centered colocated variables. International Journal of Heat and Mass Transfer 48 (2005) 1117-1127 